\title{
Quantification of Cytokinins using High-Resolution Accurate-Mass Orbitrap Mass Spectrometry and Parallel Reaction Monitoring (PRM)
}

\author{
Anna Kisiala*†, Shrikaar Kambhampati§, Naomi L. Stock $\downarrow$, Megan Aoki†, R.J. Neil Emery $\dagger$ \\ ${ }^{\dagger}$ Department of Biology, Trent University, 1600 West Bank Dr., Peterborough Ontario Canada K9L \\ 0G2, § Donald Danforth Plant Science Center, 975 N Warson Rd, Saint Louis, Missouri USA 63132, * \\ Water Quality Centre, Trent University, 1600 West Bank Dr., Peterborough Ontario Canada K9L 0G2
}

\begin{abstract}
Cytokinins (CKs) are adenine derivatives that act as phytohormones. These signaling molecules control plant cell division and differentiation, organ growth, and senescence, and they orchestrate plant interactions with biotic and abiotic environments. While CKs are predominately recognized as plant-based substances, CKs have been found across different domains of life, including microorganisms, insects, mammals, and humans. In plants, CKs act at trace, often low femtomolar concentrations; therefore, sensitive and precise analytical techniques are required to accurately detect and quantify them from complex biological matrices. Here, we report the first comprehensive CK quantification method using a QExactive Orbitrap mass spectrometer in high-resolution with a parallel reaction monitoring (PRM)-based approach. The current method progresses upon multiple reaction monitoring (MRM) methods, previously used for CK profiling on triple quadrupole mass spectrometers. This method offers improved mass accuracy and the complete product ion mass spectra (MS/MS) for compound determination with increased specificity, and sensitivity comparable with triple quadrupole instruments. The presented PRM approach was successfully applied to quantify $32 \mathrm{CKs}$ in several biological samples.
\end{abstract}



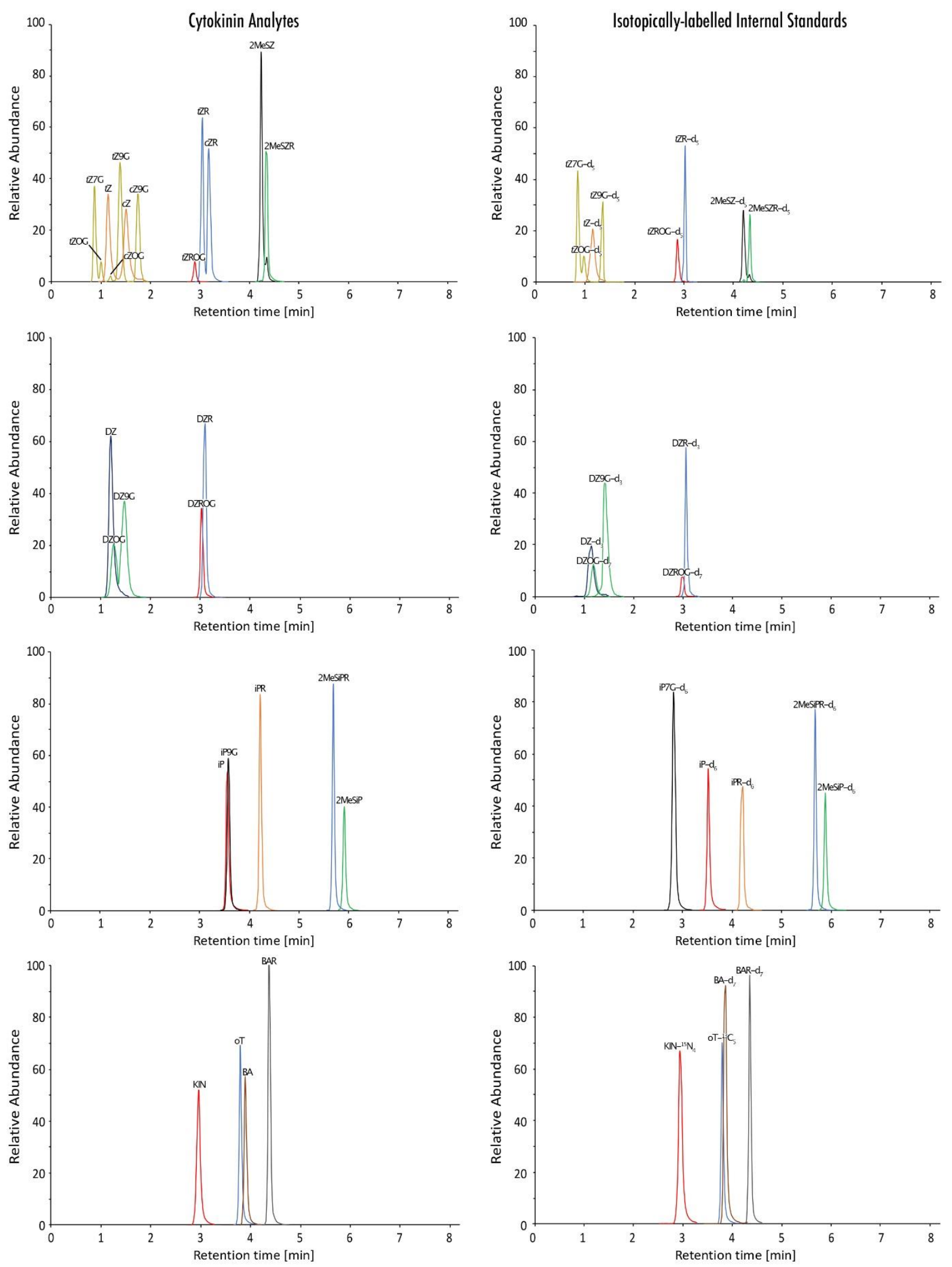

Figure S1. The extracted ion chromatograms (XIC) of zeatin (Z)-, dihydrozeatin (DZ)-, isopentenyladenine (iP)- and aromatic-type cytokinins (left), and their corresponding isotopically-labeled standards (IS; right). Authentic and labeled CK nucleotides are not included as they were analyzed in their dephosphorylated, riboside state. 

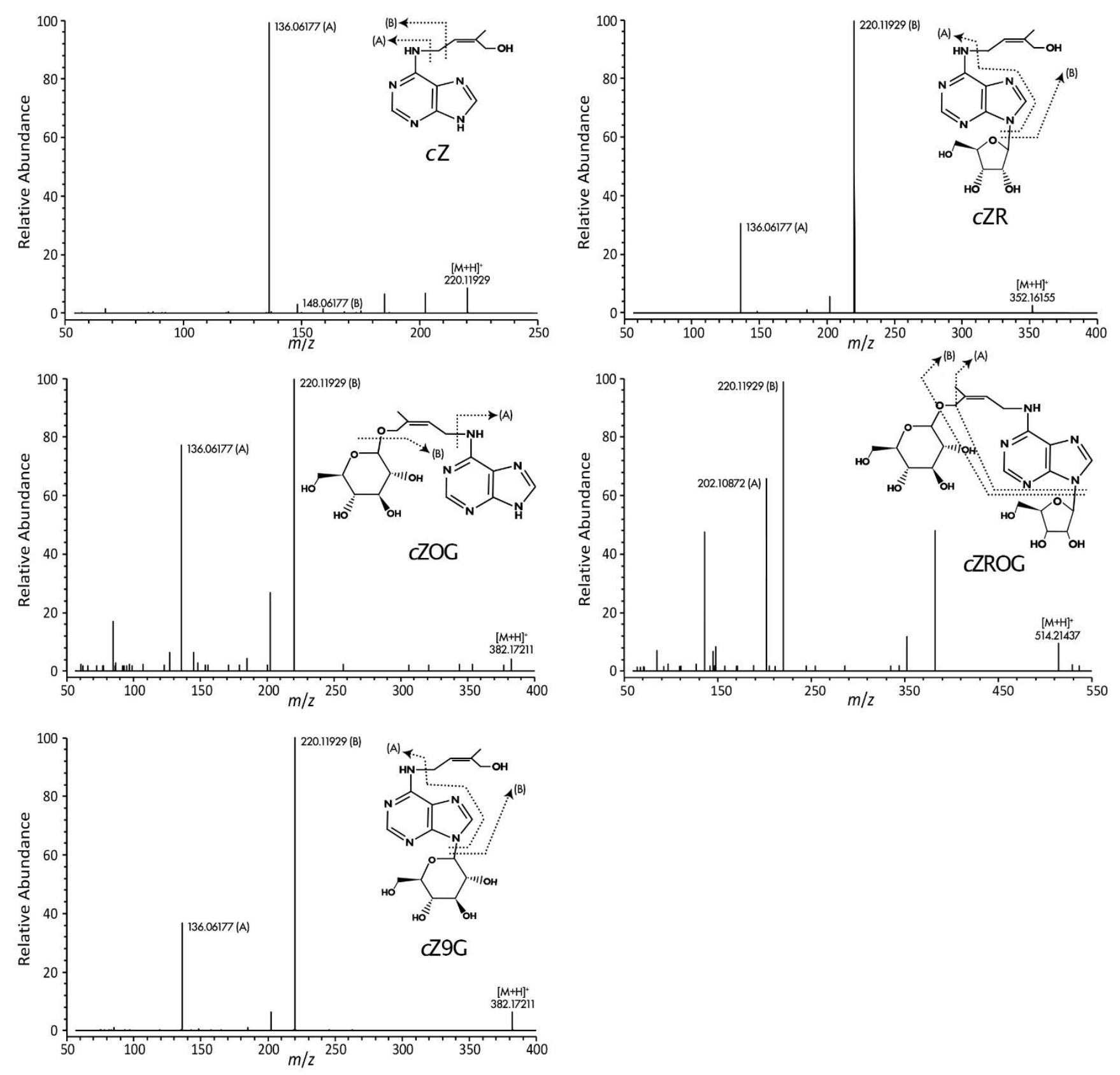

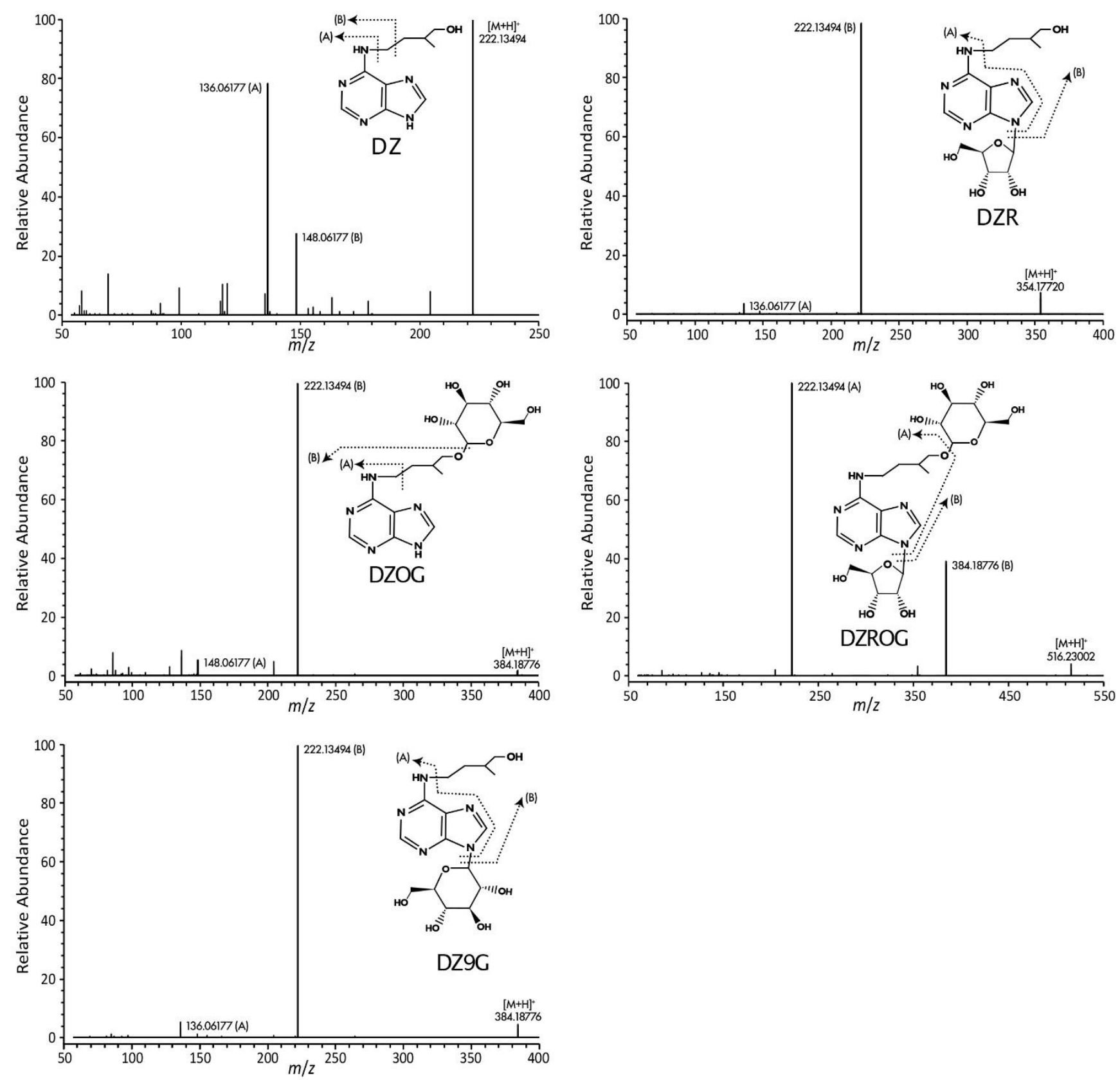

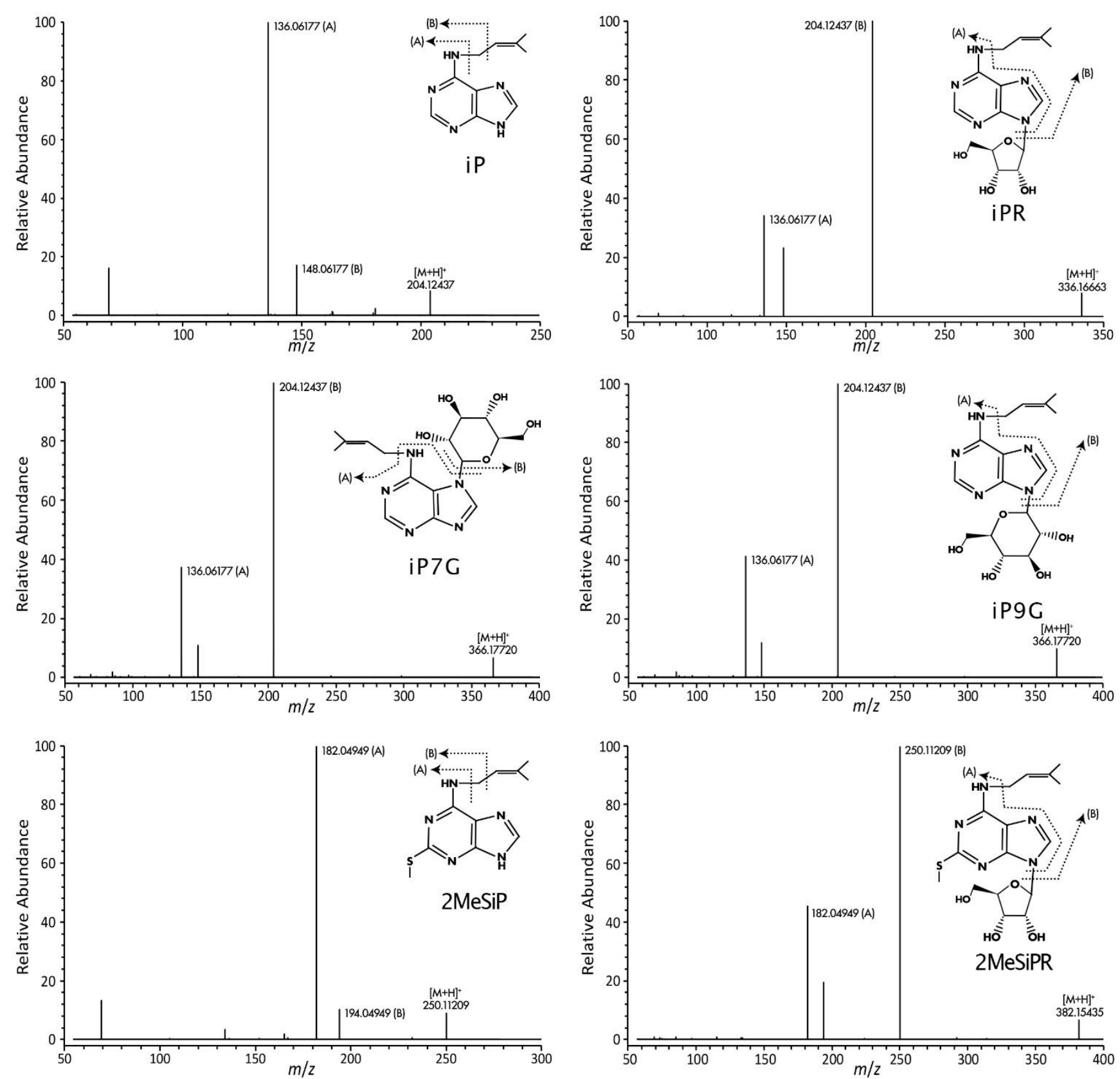

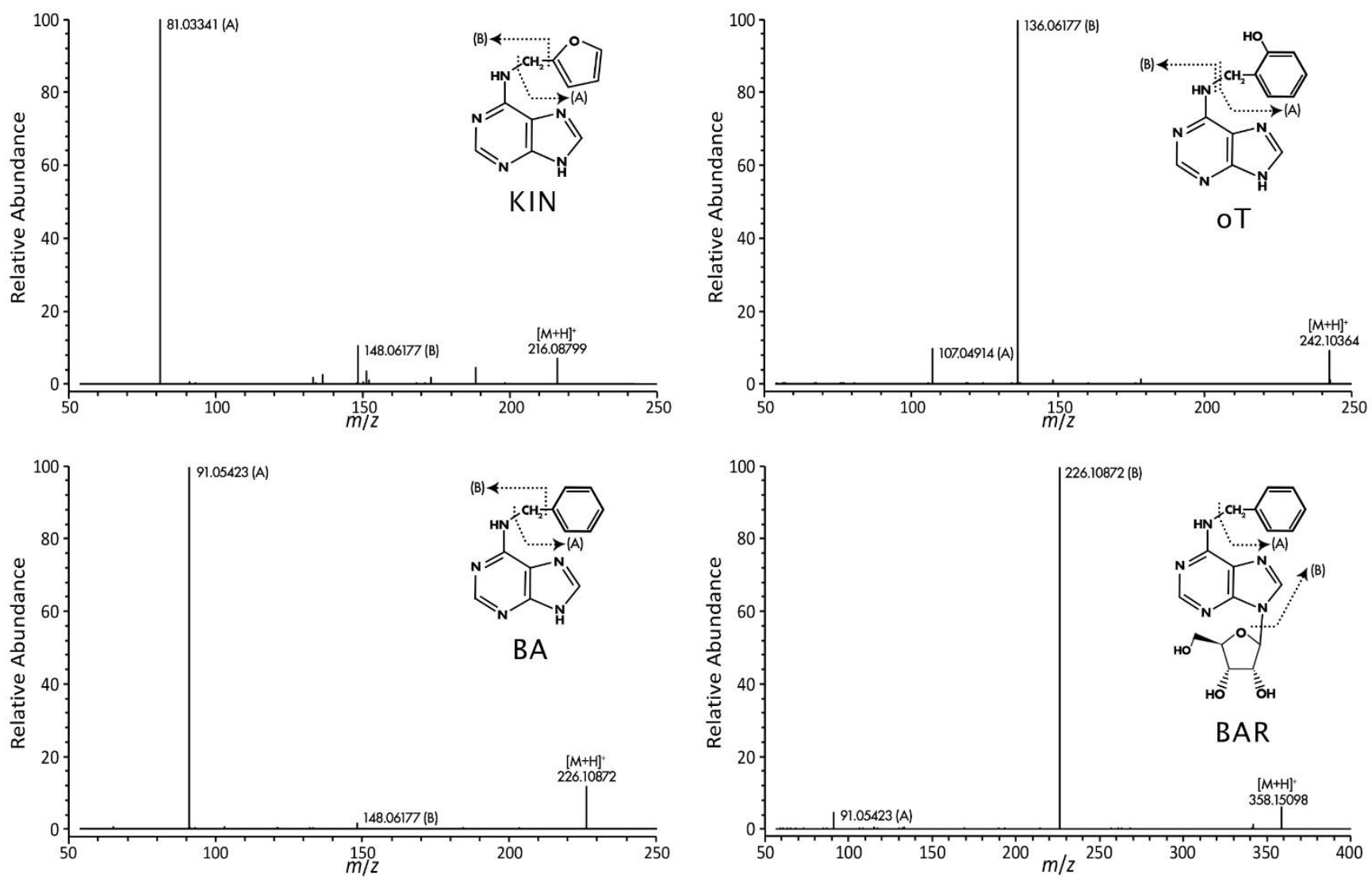

Figure S2. Chemical structures and fragmentation mass spectra of 20 cytokinins obtained using a QExactive Orbitrap in PRM mode. Arrows indicate the cleavages resulting in the two most abundant fragments (A, B). Product ions obtained from these fragmentations were used for compound quantification. $[\mathrm{M}+\mathrm{H}]^{+}-$precursor ion. Authentic and labeled CK nucleotides are not included as they were analyzed in their dephosphorylated, riboside state. 

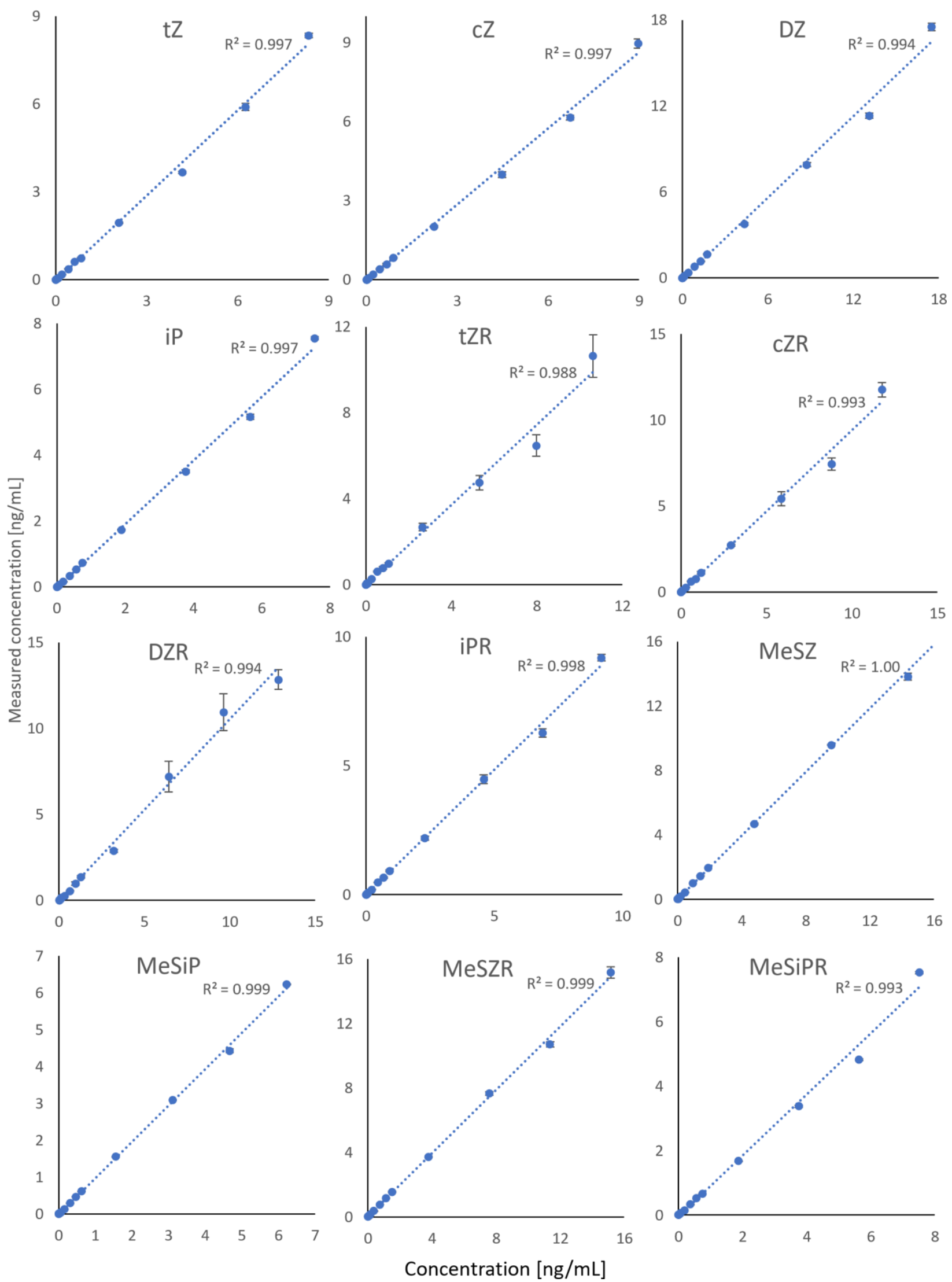

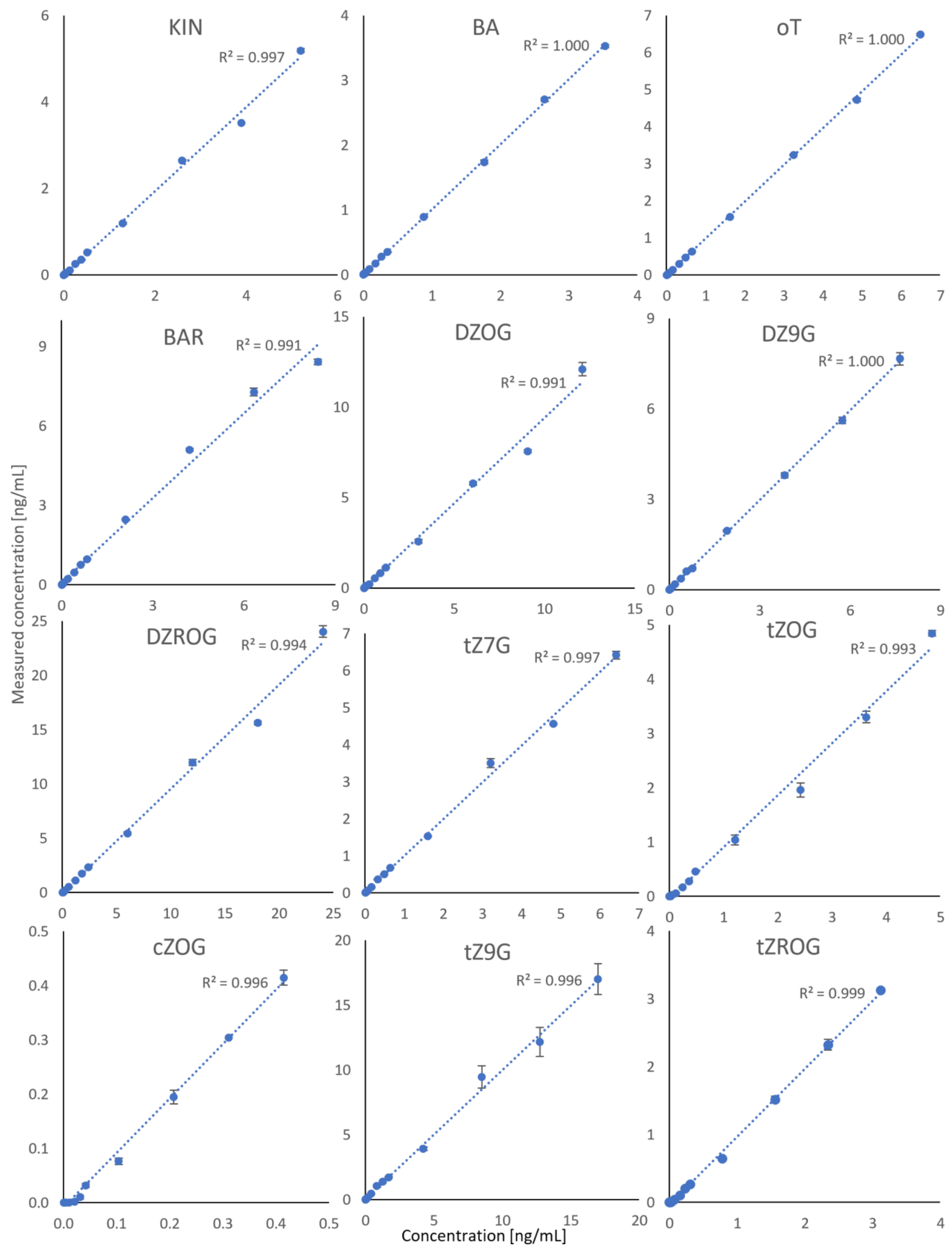


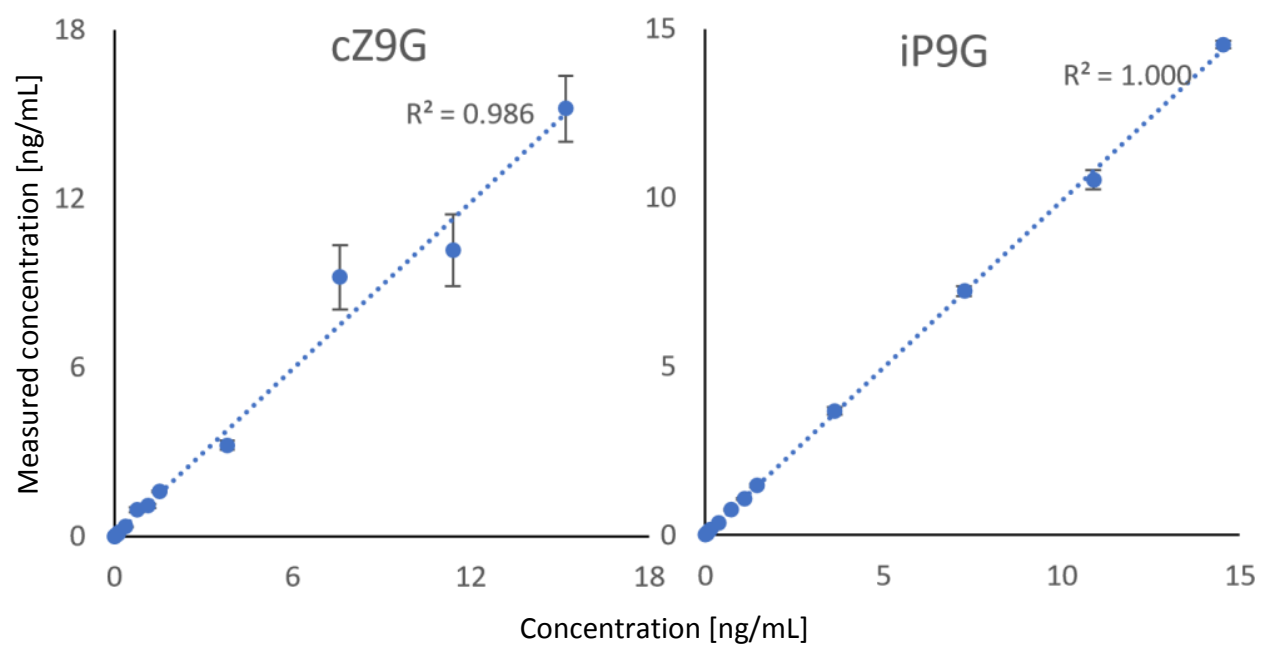

Figure S3. Linearity evaluation of the PRM method using concentration curves for the tested cytokinin analytes. iP7G and cZROG are not included and CK nucleotides were analyzed in their dephosphorylated, riboside state. 